\title{
Education modulates brain maintenance in presymptomatic frontotemporal dementia
}

Stefano Gazzina, ${ }^{1}$ Mario Grassi, ${ }^{2}$ Enrico Premi, ${ }^{3}$ Maura Cosseddu, ${ }^{4}$ Antonella Alberici, ${ }^{1}$ Silvana Archetti, ${ }^{5}$ Roberto Gasparotti, ${ }^{6}$ John van Swieten, ${ }^{7}$ Daniela Galimberti, ${ }^{8,9}$ Raquel Sanchez-Valle, ${ }^{10}$ Robert Laforce $\mathrm{Jr},{ }^{11}$ Fermin Moreno, ${ }^{12}$ Matthis Synofzik, ${ }^{13,14}$ Caroline Graff, ${ }^{15}$ Mario Masellis, ${ }^{16}$ Maria Carmela Tartaglia, ${ }^{17}$ James B Rowe, ${ }^{18}$ Rik Vandenberghe, ${ }^{19}$ Elizabeth Finger, ${ }^{20}$ Fabrizio Tagliavini, ${ }^{21}$ Alexandre de Mendonça, ${ }^{22}$ Isabel Santana, ${ }^{23}$ Chris Butler, ${ }^{24}$ Simon Ducharme, ${ }^{25,26}$ Alex Gerhard, ${ }^{27}$ Adrian Danek, ${ }^{28}$ Johannes Levin, ${ }^{28}$ Markus Otto, ${ }^{29}$ Giovanni Frisoni, ${ }^{30,31}$ Sandro Sorbi, ${ }^{32,33}$ Alessandro Padovani, ${ }^{1}$ Jonathan D Rohrer, ${ }^{34}$ Barbara Borroni, ${ }^{1 *}$ on behalf of the Genetic FTD Initiative, GENFI. ${ }^{\#}$

\section{Word count: 2648}

Number of references: 44

${ }^{1}$ Centre for Neurodegenerative Disorders, Neurology Unit, Department of Clinical and Experimental Sciences, University of Brescia, Brescia, Italy

${ }^{2}$ Department of Brain and Behavioral Science, Medical and Genomic Statistics Unit, University of Pavia, Pavia, Italy

${ }^{3}$ Stroke Unit, Neurology Unit, Spedali Civili Hospital, Brescia, Italy

${ }^{4}$ Neurology Unit, Spedali Civili Hospital, Brescia, Italy

${ }^{5}$ Biotechnology Laboratory, Department of Diagnostics, Spedali Civili Hospital, Brescia, Italy

${ }^{6}$ Neuroradiology Unit, University of Brescia, Brescia, Italy

${ }^{7}$ Department of Neurology, Erasmus Medical Center, Rotterdam, The Netherlands

8 University of Milan, Centro Dino Ferrari, Milan, Italy 
${ }^{9}$ Fondazione IRCSS Ca' Granda, Ospedale Maggiore Policlinico, Neurodegenerative Diseases Unit, Milan, Italy

${ }^{10}$ Neurology Department, Hospital Clinic, Institut d'Investigacions Biomèdiques, Barcelona, Spain

${ }^{11}$ Clinique Interdisciplinaire de Mémoire, Département des Sciences Neurologiques, $\mathrm{CHU}$ de

Québec, and Faculté de Médecine, Université Laval, Québec, Canada

12 Department of Neurology, Hospital Universitario Donostia, San Sebastian, Gipuzkoa, Spain

${ }^{13}$ Department of Neurodegenerative Diseases, Hertie-Institute for Clinical Brain Research \& Center of Neurology, University of Tübingen, Germany

${ }^{14}$ German Center for Neurodegenerative Diseases (DZNE), Tübingen, Germany

${ }^{15}$ Karolinska Institutet, Department NVS, Center for Alzheimer Research, Division of Neurogenetics, Stockholm, Sweden

${ }^{16}$ LC Campbell Cognitive Neurology Research Unit, Sunnybrook Research Institute, Toronto, Ontario, Canada

${ }^{17}$ Toronto Western Hospital, Tanz Centre for Research in Neurodegenerative Disease, Toronto, Ontario, Canada

${ }^{18}$ Department of Clinical Neurosciences, University of Cambridge, Cambridge, UK

${ }^{19}$ Laboratory for Cognitive Neurology, Department of Neurosciences, KU Leuven, Leuven, Belgium

${ }^{20}$ Department of Clinical Neurological Sciences, University of Western Ontario, London, ON, Canada

${ }^{21}$ Fondazione Istituto di Ricovero e Cura a Carattere Scientifico Istituto Neurologico Carlo Besta, Milan, Italy

${ }^{22}$ Faculty of Medicine, University of Lisbon, Lisbon, Portugal

${ }^{23}$ Neurology Department, Centro Hospitalar e Universitário de Coimbra, Coimbra, Portugal

${ }^{24}$ Department of Clinical Neurology, University of Oxford, Oxford, UK 
${ }^{25}$ Department of Psychiatry, McGill University Health Centre, McGill University, Montreal, Canada

${ }^{26}$ McConnell Brain Imaging Centre, Montreal Neurological Institute, McGill University, Montreal, Canada

${ }^{27}$ Institute of Brain, Behaviour and Mental Health, The University of Manchester, Withington, Manchester, UK

${ }^{28}$ Neurologische Klinik und Poliklinik, Ludwig-Maximilians-Universität, Munich, German Center for Neurodegenerative Diseases (DZNE), Munich, Germany

${ }^{29}$ Department of Neurology, University Hospital UIm, UIm, Germany

30 Istituto di Ricovero e Cura a Carattere Scientifico (IRCCS) Istituto Centro San Giovanni di Dio Fatebenefratelli, Brescia, Italy

${ }^{31}$ Memory Clinic and LANVIE-Laboratory of Neuroimaging of Aging, University Hospitals and University of Geneva, Geneva, Switzerland

${ }^{32}$ Department of Neuroscience, Psychology, Drug Research and Child Health, University of Florence, Florence, Italy

${ }^{33}$ Istituto di Ricovero e Cura a Carattere Scientifico (IRCCS) "Don Gnocchi”, Florence, Italy

${ }^{34}$ Dementia Research Centre, UCL Institute of Neurology, London, UK

\#List of other GENFI Consortium Members in Appendix

\section{*Corresponding author:}

Barbara Borroni, MD

Neurology Unit, University of Brescia

Piazzale Spedali Civili 1, Brescia, 25123, Italy

e-mail: bborroni@inwind.it 


\section{ABSTRACT}

Objective: Cognitively engaging lifestyles have been associated with reduced risk of conversion to dementia. Multiple mechanisms have been advocated, including increased brain volumes (i.e., brain reserve) and reduced disease progression (i.e., brain maintenance). In cross-sectional studies of presymptomatic frontotemporal dementia (FTD), higher education has been related to increased grey matter volume. Here, we examine the effect of education on grey matter loss over time.

Methods: Two-hundred twenty-nine subjects at-risk of carrying a pathogenic mutation leading to FTD underwent longitudinal cognitive assessment and T1-weighted magnetic resonance imaging at baseline and at 1-year follow-up. The first principal component score (PC1) of the Graph-Laplacian Principal Component Analysis (gLPCA) on 112 grey matter region-of-interest volumes was used to summarize the grey matter volume (GMV). The effects of education on cognitive performances and GMV at baseline and on the change between 1-year follow-up and baseline (slope) were tested by Structural Equation Modeling (SEM).

Results: Highly educated at-risk subjects had better cognition and higher grey matter volume at baseline; moreover, higher educational attainment was associated with slower loss of grey matter over time in mutation carriers.

Conclusions: This longitudinal study demonstrates that even in presence of ongoing pathological processes, education may facilitate both brain reserve and brain maintenance in the presymptomatic phase of genetic FTD.

Key words: frontotemporal dementia (FTD), brain reserve, brain maintenance, graph theory, magnetic resonance imaging (MRI) 


\section{INTRODUCTION}

Frontotemporal Dementia (FTD) is a neurodegenerative disorder characterized by executive dysfunction, personality changes and language impairment, along with atrophy of frontal and temporal lobes.[1, 2] FTD has a strong genetic background with autosomal dominant inheritance in around a third of patients. Mutations in Microtubule-Associated Protein Tau (MAPT), Granulin (GRN) and chromosome 9 open reading frame 72 (C9orf72) genes are proven major causes of genetic FTD, accounting for $10-20 \%$ of all FTD cases.[3]

There is wide variation in the age at onset within genes and within families with the same mutation, and possible disease modifiers have been recently reported. Identification of disease modifiers is key to correctly select subjects, reduce heterogeneity and increase statistical power of analysis of clinical trials, to stage presymptomatic disease and to enable long-term care planning in at-risk subjects.

Genetic variations within Transmembrane Protein 106B (TMEM106B) have been suggested to modulate disease onset in Frontotemporal Lobar Degeneration due to TAR DNA binding protein 43 proteinopathy (FTLD-TDP),[4, 5] and more recently, GDNF Family Receptor Alpha 2 (GFRA2) polymorphism and C6orf10/LOC101929163 locus have been further implied in affecting the onset in GRN and C9orf72 mutation carriers, respectively.[6, 7]

Along with non-modifiable genetic determinants, modifiable factors that modulate brain structure and function have been identified. For example, educational attainment contributes to resilience against brain damage in neurodegenerative disorders including Alzheimer's disease and FTD,[8, 9] in symptomatic and presymptomatic disease stages. In particular, it has been shown that higher educational achievements are associated with greater grey matter volumes in presymptomatic subjects carrying pathogenic FTD mutations.[10] These findings corroborated previous studies in healthy individuals, in which life exposures, such as educational and occupational attainments and 
engagement in leisure and social activities, have been associated with decreased risk of developing dementia[11, 12] and with greater brain volumes.[13, 14]

These results argue that education, a proxy measure of brain reserve, may postpone FTD symptom onset; however, these findings cannot give any information on the role of educational attainment in counteracting the effect of the pathogenic mutation on brain changes over time, i.e. actively coping with pathology progression.[15] This concept, called brain maintenance, cannot be measured through cross-sectional data, but requires longitudinal studies.[16] Indeed, if lifetime exposures, such as education, influence brain maintenance in at-risk subjects, this would have to be carefully evaluated in defining clinical trials' designs and outcomes and it might itself be considered an interventional approach.

In the present study, we aimed at evaluating the effect of educational attainment on longitudinal grey matter changes and cognitive performances in a large cohort of at-risk subjects from the Genetic FTD Initiative (GENFI) study. 


\section{METHODS}

\subsection{Participants}

Data for this study were drawn from the GENFI multicentre cohort study, which consists of 27 research centres across Europe and Canada (www.genfi.org.uk). For the purpose of the present study, we included subjects at-risk of carrying mutations in C9orf72, MAPT and GRN, as having the proband with monogenic FTD[17] and for whom both baseline and 1-year follow-up magnetic resonance imaging (MRI) was available. Conversion to symptomatic stage at follow-up visit or the presence of psychiatric disease or central nervous system pathology, including expansive or vascular lesions, were considered exclusion criteria.

Between January 2012 and December 2017, 229 at-risk subjects fulfilled inclusion/exclusion criteria, namely 116 mutation carriers (C9orf72 $\mathrm{n}=31 ; G R N \mathrm{n}=65 ;$ MAPT $\mathrm{n}=20$ ) and 113 mutation non-carriers.

Local ethics committees approved the study at each site and all participants provided written informed consent; the study was conducted according to the Declaration of Helsinki. For each subject we recorded demographic data, including years of formal schooling (education), past medical history, and a standardized clinical and neuropsychological assessment, as previously published.[17] We considered education as reserve proxy and Mini-Mental examination (MMSE) raw scores as measure of cognitive status.

Furthermore, we considered age, sex and TMEM106B genotype (see[10] for details), as variables of interest in the statistical model.

\section{MRI processing}

Participants were scanned at their local site on scanners from three different manufacturers (Philips Healthcare, GE Healthcare Life Sciences, Siemens Healthcare Diagnostics). Magnetic field 
strength was 3T for 221 scans (96.5\%) and 1.5T for 8 scans (3.5\%). The protocol, designed to match across scanners as much as possible, included a volumetric T1-weighted MRI scan, as previously published.[17]

Baseline and follow-up scans were processed using the standardized longitudinal voxel-based morphometry pipeline of the Computational Anatomy Toolbox (CAT v.12.1, extension to SPM12 v.7219 running on MATLAB R2015a) (http://www.neuro.uni-jena.de/cat/).

Baseline and follow-up grey matter volume (GMV) maps were parcelled into 112 cortical and subcortical regions (excluding the cerebellum because of some subjects with incomplete coverage of the inferior cerebellar hemispheres[18]) according to the maximum probability tissue labels derived from the "MICCAI 2012 Grand Challenge and Workshop on Multi-Atlas Labeling" (https://my.vanderbilt.edu/masi/workshops/). This atlas was created from MRI scans belonging to the OASIS project (www.oasis-brains.org/) and labels were provided by Neuromorphometrics, Inc. (www.neuromorphometrics.com/).

Tissue volumes were estimated in the native space, before any spatial normalization. Thus, region of interests (ROIs) values, representing the GMV contained in each ROI (expressed in milliliters, $\mathrm{mL}$ ), were further corrected for the total intracranial volume (TIV). Estimates of TIV, total GMV, total white matter volume (WMV) and total cerebrospinal fluid volume (CSFV) were also computed to assess macroscopic differences. Total GMV, total WMV and total CSFV were expressed as percentage of TIV.

\subsection{Statistical analysis}

To overcome the complexity of MRI data, graph-Laplacian Principal Component Analysis (gLPCA) was applied to obtain a low dimensional representation of grey matter parcellation at baseline and at follow-up,[10] which incorporated graph structure. gLPCA has several advantages 
compared to Principal Component Analysis (PCA): (i) it is modelled on the representation of the data; (ii) it can be easily calculated, presenting a compact closed-form solution; and (iii) it allows noise removal. The first principal component score (PC1) was used to summarise GMV at each time point. A correlation threshold higher than 0.6 was used to define PC1, which was constituted by 100 ROIs belonging to frontal, cingulate, temporal and parietal regions.

Successively, a two-group Structural Equation Model (SEM) was fitted on longitudinal data. SEM is a multivariate regression technique that models the covariance structure of a set of observed and latent (random effects) variables, and is based on a subset of possible paths connecting those variables, incorporating directional information (regression coefficients) and bidirectional information (covariance).

The study design was reported in Figure 1. We considered mutation carriers and mutation noncarriers separately. In the two groups, the effect of education was evaluated on a) cognitive performances (as measured by MMSE) at baseline; b) GMV (as measured by PC1) at baseline; c) the slope of cognitive performances between 1-year follow-up and baseline; $d$ ) the slope of GMV between 1-year follow-up and baseline. Moreover, we evaluated the effect of e) GMV at baseline on the cognitive performances at baseline, f) GMV at baseline on the slope of cognitive performances; g) the slope of GMV on the slope of cognitive performances; $h$ ) the cognitive performances at baseline on the slope of GMV. Finally, we evaluated the covariance between i) the baseline and the slope of cognitive performances, and j) the baseline and the slope of GMV. Regression effects were adjusted by observed covariates, namely age and sex; in view of previous evidence for TMEM106B polymorphism effect on GMV in presymptomatic mutation carriers,[10] we also considered TMEM106B genotype (rs1990622 T/T, T/C, C/C, recorded using addictive coding $0,1,2)$, as covariate. 
We did not include random effects (latent covariates), such as family's pedigree and Country, on the basis of an initial exploratory analysis that indicated no significant effects of these variables.

\section{Figure 1 about here.}

Baseline and follow-up demographic, cognitive and volumetric variables were compared across groups using independent t-test or paired sample t-test for continuous variables and Fisher's exact tests for dichotomous variables. Exploratory Random Effect Models was performed by "Ime4" R package. SEM analysis was performed via "lavaan" R package, using Full Information Maximum Likelihood Method (FIML) for simultaneously estimating SEM parameters and imputing MMSE score and TMEM106B genotype random missing values. In addition, for quality control, MMSE score and TMEM106B genotype missing values were imputed with Nonparametric Random Forest Imputation procedure of the "missForest" R package, and imputed data matrix was successively used for SEM analysis. Two-group SEM analysis was performed by an overall Likelihood Ratio Test (LRT) of two SEM models: model (1) with unequal regression coefficients, and residual (co)variances in the two groups vs. model (0) with equal regression coefficients, and residual (co)variances. Finally, a model (2) was fitted considering the group as covariate and adding the interaction terms education* group and $T M E M^{*}$ group, for evaluating the statistical significance of the regression coefficient differences between the two-groups. P-values less than 0.05 were considered significant. 


\section{RESULTS}

Demographic characteristics of at-risk asymptomatic subjects, i.e. mutation carriers and mutation non-carriers, are reported in Table 1. Non-carriers were older than carriers $(p=0.036)$; no other significant differences were found in sex, years of schooling, MMSE at baseline and brain volumes at baseline between groups. No significant group-wise differences were found in MMSE and brain volumes changes at 1-year follow-up in either carriers or non-carriers. 
Table 1. Demographical characteristics and brain volumes of the cohort.

\begin{tabular}{lccc}
\hline Variables & $\begin{array}{c}\text { Mutation } \\
\text { carriers }\end{array}$ & $\begin{array}{c}\text { Mutation } \\
\text { non- } \\
\text { carriers }\end{array}$ & p-value $^{\wedge}$ \\
\hline $\mathrm{N}^{\circ}$ of subjects & 116 & 113 & - \\
\hline All & 26.7 & - & \\
\hline C9orf72, \% & 56.0 & - & \\
\hline GRN, \% & 17.2 & - & \\
\hline MAPT, \% & 60.3 & 58.4 & n.s. ${ }^{\dagger}$ \\
\hline Sex, female \% & $14.4 \pm 3.4$ & $14.0 \pm 3.2$ & n.s. \\
\hline Education, years & $45.7 \pm 11.2$ & $49.2 \pm 14.0$ & 0.036 \\
\hline Age at baseline visit, years & $47.1 \pm 11.3$ & $50.6 \pm 14.1$ & 0.038 \\
\hline Age at follow-up visit, years & $-12.1 \pm 11.5$ & - & - \\
\hline Expected age at onset, years* & $29.4 \pm 1.2$ & $29.4 \pm 0.9$ & n.s. \\
\hline MMSE, baseline & $29.3 \pm 1.1$ & $29.4 \pm 1.0$ & n.s. \\
\hline MMSE, follow-up & $1498 \pm 151$ & $1490 \pm 123$ & n.s. \\
\hline TIV baseline, mL & $1500 \pm 141$ & $1492 \pm 128$ & n.s. \\
\hline TIV follow-up, $\mathrm{mL}$ & $42.8 \pm 3.5$ & $42.7 \pm 3.7$ & n.s. \\
\hline Total GMV baseline, \% & $42.6 \pm 3.7$ & $42.6 \pm 3.6$ & n.s. \\
\hline Total GMV follow-up, \% & $34.0 \pm 2.5$ & $33.6 \pm 2.5$ & n.s. \\
\hline Total WMV baseline, \% & $33.7 \pm 2.5$ & $33.6 \pm 2.7$ & n.s. \\
\hline Total WMV follow-up, \% & $23.1 \pm 4.8$ & $23.7 \pm 4.8$ & n.s. \\
\hline Total CSFV baseline, \% & $23.7 \pm 4.9$ & $23.9 \pm 4.9$ & n.s. \\
\hline Total CSFV follow-up, \% & & & \\
\hline
\end{tabular}

$\wedge$ Two sample t-test, otherwise specified: †Fisher's exact test.

$p$ refers to mutation carriers $v s$. mutation non-carriers comparisons; no significant differences between baseline vs. follow-up MMSE scores and brain volumes in both mutation non-carriers and in mutation carriers were found. Results are expressed as mean \pm standard deviation, unless otherwise specified.

MMSE: Mini-Mental State Examination; TIV: total intracranial volume; GMV: grey matter volume; WMV: white matter volume; CSFV: cerebrospinal fluid volume; n.s.: not significant.

*computed as previously published [17] 
SEM fitting results are shown in Table 2 and Figure 2. Overall, the two-group models' difference was statistically significant $(\mathrm{LRT}=34.3, \mathrm{df}=20, \mathrm{p}$-value=0.019).

In mutation carriers, significant direct effects of education on cognitive performances (as measured by MMSE) and on GMV at baseline (as measured by PC1, which summarised ROI measures) were found (beta $=0.349,95 \% \mathrm{Cl}=0.047$ to $0.650, \mathrm{p}=0.023$ and beta $=0.284,95 \% \mathrm{Cl}=0.047$ to $0.521, p=0.019$, respectively). Moreover, education had a significant inverse effect on GMV slope (beta $=-0.270,95 \% \mathrm{Cl}=-0.501$ to $-0.041, p=0.021$ ), the higher the years of formal schooling the lower the loss of GMV at follow-up.

No significant effect of education on cognitive performances' slope at 1-year follow-up was observed (beta $=0.125,95 \% \mathrm{Cl}=-0.174$ to $0.423, p=0.413)$. No direct effect $(p>0.05)$ between baseline and slopes of cognitive performances and GMV was observed, while expected significant negative covariances were confirmed (cov $=-0.636,95 \% \mathrm{Cl}=-0.869$ to $-0.402, p<0.001$ and $\mathrm{cov}=-0.305,95 \% \mathrm{Cl}=-0.444$ to $-0.166, \mathrm{p}<0.001$ for cognitive performances and $\mathrm{GMV}$, respectively). These above effects were similarly present in non-carriers, with the distinctive difference for the null effect of education on GMV slope (beta $=-0.020,95 \% \mathrm{Cl}=-0.181$ to $0.140, p=0.806$ ). Notably, in mutation non-carriers, the significant direct effect of education on cognitive performances was greater (two-fold) than in mutation carriers (beta $=0.548,95 \% \mathrm{Cl}=0.289$ to $0.807, \mathrm{p}<0.001$ ). Nevertheless, the two-group beta differences (the two-way interaction effect) was statistically suggestive in the combined group SEM analysis $(p=0.088)$. In addition, a significant covariate effect of TMEM106B genotype was observed in mutation carriers, and it was not shown in mutation non-carriers (the two-way interaction testing was statistically significant: $p=0.041$ ), confirming the previous evidence [10] of the modulating effect of TMEM106B genotype on GMV in presymptomatic FTD (beta $=0.468,95 \% \mathrm{Cl}=0.189$ to 0.747 , 
$p=0.001$ and beta $=0.034,95 \% \mathrm{Cl}=-0.119$ to $0.186, p=0.665$ for mutation carriers and non-carriers, respectively). 
Table 2. Structural Equation Model in mutation carriers and mutation non-carriers.

\begin{tabular}{|c|c|c|c|c|c|c|c|c|}
\hline \multirow[t]{2}{*}{ Variable } & \multicolumn{4}{|c|}{ Mutation carriers } & \multicolumn{4}{|c|}{ Mutation non-carriers } \\
\hline & Estimate & SE & z-value & p-value & Estimate & SE & z-value & p-value \\
\hline \multicolumn{9}{|l|}{ MMSE, baseline } \\
\hline GMV baseline & 0.074 & 0.115 & 0.644 & 0.520 & -0.025 & 0.110 & -0.231 & 0.817 \\
\hline Sex & -0.003 & 0.208 & -0.014 & 0.989 & -0.058 & 0.163 & -0.355 & 0.723 \\
\hline Age & -0.160 & 0.100 & -1.599 & 0.110 & 0.006 & 0.076 & 0.078 & 0.938 \\
\hline TMEM106B & 0.147 & 0.184 & 0.797 & 0.425 & 0.058 & 0.129 & 0.447 & 0.655 \\
\hline Education & 0.349 & 0.153 & 2.279 & 0.023 & 0.548 & 0.132 & 4.145 & $<0.001$ \\
\hline \multicolumn{9}{|l|}{ MMSE, slope } \\
\hline GMV baseline & 0.092 & 0.122 & 0.748 & 0.454 & 0.101 & 0.153 & 0.661 & 0.509 \\
\hline GMV slope & 0.141 & 0.109 & 1298 & 0.194 & 0.123 & 0.185 & 0.668 & 0.504 \\
\hline Sex & 0.117 & 0.208 & 0.561 & 0.575 & -0.370 & 0.207 & -1.787 & 0.074 \\
\hline Age & 0.098 & 0.102 & 0.961 & 0.336 & -0.200 & 0.099 & -2.013 & 0.044 \\
\hline TMEM106B & 0.086 & 0.182 & 0.470 & 0.639 & -0.249 & 0.162 & -1.533 & 0.125 \\
\hline Education & 0.125 & 0.152 & 0.818 & 0.413 & -0.307 & 0.166 & -1.849 & 0.065 \\
\hline \multicolumn{9}{|l|}{ GMV, baseline } \\
\hline Sex & 0.104 & 0.168 & 0.619 & 0.536 & -0.020 & 0.140 & -0.145 & 0.884 \\
\hline Age & -0.386 & 0.072 & -5.333 & $<0.001$ & -0.428 & 0.051 & -8.424 & $<0.001$ \\
\hline TMEM106B & 0.468 & 0.142 & 3.287 & 0.001 & 0.086 & 0.110 & 0.778 & 0.437 \\
\hline Education & 0.284 & 0.121 & 2.347 & 0.019 & 0.277 & 0.110 & 2.515 & 0.012 \\
\hline \multicolumn{9}{|l|}{ GMV, slope } \\
\hline MMSE baseline & -0.043 & 0.064 & -0.664 & 0.507 & -0.017 & 0.049 & -0343 & 0.731 \\
\hline Sex & -0.357 & 0.160 & -2.235 & 0.025 & -0.178 & 0.098 & -1.814 & 0.070 \\
\hline Age & -0.060 & 0.070 & -0.857 & 0.392 & -0.009 & 0.036 & -0.256 & 0.798 \\
\hline TMEM106B & -0.072 & 0.136 & -0.582 & 0.597 & 0.034 & 0.078 & 0.433 & 0.665 \\
\hline Education & -0.270 & 0.117 & -2.303 & 0.021 & -0.020 & 0.082 & -0.246 & 0.806 \\
\hline \multicolumn{9}{|l|}{ Covariances } \\
\hline $\begin{array}{l}\text { MMSE baseline with } \\
\text { MMSE slope }\end{array}$ & -0.636 & 0.119 & 5.340 & $<0.001$ & -0.514 & 0.096 & -5.375 & $<0.001$ \\
\hline $\begin{array}{l}\text { GMV baseline with GMV } \\
\text { slope }\end{array}$ & -0.305 & 0.071 & 4.309 & $<0.001$ & $-0-186$ & 0.038 & -4.860 & $<0.001$ \\
\hline
\end{tabular}

MMSE: Mini-mental examination test; GMV: grey matter volume as measured by first component (PC1, see text for details); SE: Standard Error; z-value= Estimate/SE.

Significant results of educational attainment's effect in boldface.

Figure 2 about here. 


\section{DISCUSSION}

Genetic FTD is preceded by a long period in which, despite the evidence of initial changes in biomarkers and brain structure, behaviour and cognition are spared.[17, 19-21] Pharmacological and non-pharmacological interventions may provide better clinical outcomes if applied in this phase, when the brain can still cope with pathology processes, and such treatments may eventually delay disease onset.[22] Beyond future disease-modifying drugs,[23] the possibility to intervene on environment and other modulating factors is attractive. Some evidence shows that cognitive stimulating environments lead to brain volumetric advantages and better cognitive performances. These effects are common to physiological[24-26] and initial pathological ageing,[27-29] suggesting that neuroplasticity is maintained even in diseased brains, regardless of the specific clinical picture or the underlying pathological process.

Two alternate hypotheses address this issue. First, that lifestyle acts passively by increasing brain volume, but does not influence on brain loss; second, lifestyle acts by increasing brain maintenance. To test the latter hypothesis longitudinal data is required. These positive effects may diminish as disease progresses to the symptomatic phase. If this second hypotheses were the case, it would be plausible to think of modulating the disease course of dementing disorders by enrichment of lifetime exposures.

In the current longitudinal study, we applied SEM analysis to test these hypotheses in presymptomatic monogenic FTD, evaluating the effect of the educational level on two outcome measures of reserve: cognitive performances and grey matter volumes. Our results seem to confirm the latter hypothesis, showing that higher education confers higher grey matter volumes and greater brain maintenance over time. Additionally, as previously reported, $[10,30]$ TMEM106B genotype significantly modulates grey matter volume at baseline in mutation carriers. 
These findings are in line with previous longitudinal studies demonstrating that reserve proxies are associated with reduced rate of hippocampal atrophy,[31, 32] reduced rate of brain hypometabolism[33] and cerebrospinal fluid biomarkers changes[33] in healthy agers and Alzheimer's disease.

One intriguing aspect of brain maintenance is that it may reflect differences in the accumulation of pathology-related changes. $[34,35]$ Such demonstration in FTD requires in vivo pathological markers (i.e. tau or TDP-43 tracers), which are not currently available.[36] This neuroprotective effect may be related to changes at the molecular level, such as increased levels of neurotrophic factors[37] and glutamate neurotransmission, [38] or at the cellular level, with increased neurogenesis,[39] synaptogenesis[40] and angiogenesis,[41] and might be able to go beyond the underlying pathogenic mechanisms related to the specific mutation ( $G R N, C 9 O R F 72, M A P T)$ or to specific proteinopathy (i.e. TDP-43 or tau).

Interestingly, as previously reported,[10] years of education had a significant effect on grey matter volume even in mutation non-carriers, supporting the idea of a generalizable beneficial effect of education. Conversely, in the present work, we did not find any effect of education on brain maintenance in mutation non-carriers, but we recognize that this could be likely due to the low variance of grey matter volume within 1-year follow-up in healthy subjects. However, longer follow-up is necessary to draw definitive conclusions.

Regarding cognition, higher education led to better cognitive performances at baseline, but not to significant effects on cognitive decline. This effect was comparable in mutation carriers and mutation non-carriers; of note, in subjects without pathogenic mutations, the beneficial effect of education on cognitive performances was greater than in mutation carriers.

We acknowledge that this study entails some limitations. Despite that education represents an environmental factor, it is often immutable because acquired in childhood/young adulthood. Thus, 
the present results do not allow to directly conclude that interventional trials could delay disease onset. However, education is known to influence professional attainment, which has been already proven a proxy measure of reserve in FTD. $[9,42]$ Also, we chose MMSE as a global measure of cognition, acknowledging that MMSE is affected only close to disease onset[17] and that it does not represent the best measure of severity even in symptomatic phases.[43] Thus, the effect of more sensitive neuropsychological tests[17] has to be evaluated in future studies, especially to assess changes of cognitive performances over time. Moreover, we could not test the effect of educational attainment in each mutation due to low sample number: larger samples are needed to address this issue. Last, due to the observational nature of the study, data on possible confounders, such as concomitant vascular risk factors, were not available. However, in a recent large-scale Mendelian randomization study of the related condition, i.e. amyotrophic lateral sclerosis, the authors confirmed educational attainment to be an important modulator based on genetics.[44]

In conclusion, these findings extend our knowledge of the reserve theory, demonstrating that in presymptomatic FTD the rate of atrophy was influenced by the educational level, with reduced grey matter loss in more educated subjects. Thus, even in presence of an ongoing pathological process, presymptomatic FTD subjects still maintain a high-performing reserve like in healthy brains, virtually turning back the clock of the disease natural history. The demonstration that differences in early lifestyle may slow down later disease progression suggests that even in monogenic disorders, outcomes are not wholly determined from birth, and this opens exciting perspectives for eventually delaying symptom onset. Future confirmatory studies assessing the role of other reserve proxies and their effect on longitudinal brain changes in symptomatic monogenic and sporadic FTD are needed. 


\section{ACKNOWLEDGEMENTS}

James B Rowe is supported by the Wellcome Trust (103838) and the Cambridge NIHR Biomedical Research Centre.

CONTRIBUTIONSHIP. Stefano Gazzina planned the study, conducted the statistical analyses, contributed to interpretation of the results, and drafted the initial version of the manuscript; Mario Grassi carried out the statistical analyses and contributed to interpretation of the results; Enrico Premi, Maura Cosseddu, Antonella Alberici, Silvana Archetti, Roberto Gasparotti, John van Swieten, Daniela Galimberti, Raquel Sanchez-Valle, Robert Laforce Jr, Fermin Moreno, Matthis Synofzik, Caroline Graff, Mario Masellis, Maria Carmela Tartaglia, James B Rowe, Rik Vandenberghe, Elizabeth Finger, Fabrizio Tagliavini, Alexandre de Mendonça, Isabel Santana, Chris Butler, Simon Ducharme, Alex Gerhard, Adrian Danek, Johannes Levin, Markus Otto, Giovanni Frisoni, Sandro Sorbi, Alessandro Padovani, and Jonathan D Rohrer contributed to acquisition of data; Barbara Borroni planned the study, contributed to interpretation of the results, drafted the initial version of the manuscript and is responsible for the overall content as guarantor.

FUNDING STATEMENT. This research did not receive any specific grant from funding agencies in the public, commercial, or not-for-profit sectors.

COMPETING of INTERESTS. Authors have no competing of interests.

ETHICS APPROVAL. The study was approved by Brescia Hospital Ethics Committee (NP2224). Local ethics committees approved the study at each site. 


\section{APPENDIX}

\section{List of other GENFI consortium members}

Maria Rosario Almeida - Centre of Neurosciences and Cell Biology, Universidade de Coimbra, Coimbra, Portugal

Sarah Anderl-Straub - Ulm University, Ulm, Germany

Christin Andersson - Department of Clinical Neuroscience, Karolinska Institutet, Stockholm, Sweden

Anna Antonell - Alzheimer's disease and other cognitive disorders unit, Neurology Department, Hospital Clinic, Institut d'Investigacions Biomèdiques, Barcelona, Spain

Andrea Arighi - Fondazione IRCSS Ca' Granda, Ospedale Maggiore Policlinico, Neurodegenerative Diseases Unit, Milan, Italy

- Mircea Balasa - Alzheimer's disease and other cognitive disorders unit, Neurology Department, Hospital Clinic, Institut d'Investigacions Biomèdiques, Barcelona, Spain

Myriam Barandiaran - Neuroscience Area, Biodonostia Health Research Institute, Paseo Dr Begiristain sn, CP 20014, San Sebastian, Gipuzkoa, Spain

- Nuria Bargalló - Radiology Department, Image Diagnosis Center, Hospital Clínic and Magnetic Resonance Image core facility, IDIBAPS, Barcelona, Spain

- Robart Bartha - Department of Medical Biophysics, Robarts Research Institute, University of Western Ontario, London, Ontario, Canada

Benjamin Bender - Department of Radiology, University of Tuebingen, Tuebingen, Germany

Luisa Benussi - Istituto di Ricovero e Cura a Carattere Scientifico Istituto Centro San Giovanni di Dio Fatebenefratelli, Brescia, Italy

Giuliano Binetti - Istituto di Ricovero e Cura a Carattere Scientifico Istituto Centro San Giovanni di Dio Fatebenefratelli, Brescia, Italy 
Sandra Black - LC Campbell Cognitive Neurology Research Unit, Sunnybrook Research Institute, Toronto, Canada

- Martina Bocchetta - Department of Neurodegenerative Disease, UCL Institute of Neurology, London, UK

Sergi Borrego-Ecija - Alzheimer's disease and other cognitive disorders unit, Neurology Department, Hospital Clinic, Institut d'Investigacions Biomèdiques, Barcelona, Spain Jose Bras - Department of Neurodegenerative Disease, UCL Institute of Neurology, London, UK - Rose Bruffaerts - Laboratory for Cognitive Neurology, Department of Neurosciences, KU Leuven, Leuven, Belgium

- Paola Caroppo - Fondazione Istituto di Ricovero e Cura a Carattere Scientifico Istituto Neurologico Carlo Besta, Milan, Italy

- David Cash - Department of Neurodegenerative Disease, UCL Institute of Neurology, London, UK Miguel Castelo-Branco - Neurology Department, Centro Hospitalar e Universitário de Coimbra, Instituto de Ciências Nucleares Aplicadas à Saúde (ICNAS), Coimbra, Portugal Rhian Convery - Department of Neurodegenerative Disease, UCL Institute of Neurology, London, UK

. Thomas Cope - University of Cambridge, Cambridge, UK

María de Arriba - Neuroscience Area, Biodonostia Health Research Institute, Paseo Dr Begiristain sn, CP 20014, San Sebastian, Gipuzkoa, Spain

- Giuseppe Di Fede - Fondazione Istituto di Ricovero e Cura a Carattere Scientifico Istituto Neurologico Carlo Besta, Milan, Italy

Zigor Díaz - ITA Alzheimer, San Sebastian, Spain

Katrina M Dick - Department of Neurodegenerative Disease, UCL Institute of Neurology, London, UK 
. Diana Duro - Faculty of Medicine, Universidade de Coimbra, Coimbra, Portugal

- Chiara Fenoglio - University of Milan, Centro Dino Ferrari, Milan, Italy

Carlos Ferreira - Instituto Ciências Nucleares Aplicadas à Saúde, Universidade de

Coimbra, Coimbra, Portugal

Catarina B. Ferreira - Faculty of Medicine, University of Lisbon, Lisbon, Portugal

Toby Flanagan - University of Manchester, Manchester, UK

Nick Fox - Department of Neurodegenerative Disease, UCL Institute of Neurology, London, UK

Morris Freedman - Division of Neurology, Baycrest Centre for Geriatric Care, University of Toronto, Toronto, Canada

Giorgio Fumagalli - Fondazione IRCSS Ca' Granda, Ospedale Maggiore Policlinico, Neurodegenerative Diseases Unit, Milan, Italy; Department of Neuroscience, Psychology, Drug Research and Child Health, University of Florence, Florence, Italy

Alazne Gabilondo - Neuroscience Area, Biodonostia Health Research Institute, Paseo Dr Begiristain sn, CP 20014, San Sebastian, Gipuzkoa, Spain

Serge Gauthier - Department of Neurology and Neurosurgery, McGill University, Montreal, Québec, Canada

Roberta Ghidoni - Istituto di Ricovero e Cura a Carattere Scientifico Istituto Centro San Giovanni di Dio Fatebenefratelli, Brescia, Italy

- Giorgio Giaccone - Fondazione Istituto di Ricovero e Cura a Carattere Scientifico Istituto Neurologico Carlo Besta, Milan, Italy

Ana Gorostidi - Neuroscience Area, Biodonostia Health Research Institute, Paseo Dr Begiristain sn, CP 20014, San Sebastian, Gipuzkoa, Spain

Caroline Greaves - Department of Neurodegenerative Disease, UCL Institute of Neurology, London, UK 
. Rita Guerreiro - Department of Neurodegenerative Disease, UCL Institute of Neurology, London, UK

. Carolin Heller - Department of Neurodegenerative Disease, UCL Institute of Neurology, London, UK Tobias Hoegen - Department of Neurology, Ludwig-Maximilians-University of Munich, Munich, Germany

Begoña Indakoetxea - Cognitive Disorders Unit, Department of Neurology, Donostia University Hospital, Paseo Dr Begiristain sn, CP 20014, San Sebastian, Gipuzkoa, Spain

Vesna Jelic - Division of Clinical Geriatrics, Karolinska Institutet, Stockholm, Sweden

Lize Jiskoot - Department of Neurology, Erasmus Medical Center, Rotterdam, The Netherlands Hans-Otto Karnath - Section of Neuropsychology, Department of Cognitive Neurology, Center for Neurology \& Hertie-Institute for Clinical Brain Research, Tübingen, Germany

Ron Keren - University Health Network Memory Clinic, Toronto Western Hospital, Toronto, Canada Maria João Leitão - Centre of Neurosciences and Cell Biology, Universidade de Coimbra, Coimbra, Portugal

Albert Lladó - Alzheimer's disease and other cognitive disorders unit, Neurology Department, Hospital Clinic, Institut d'Investigacions Biomèdiques, Barcelona, Spain

Gemma Lombardi - Department of Neuroscience, Psychology, Drug Research and Child Health, University of Florence, Florence, Italy

Sandra Loosli - Department of Neurology, Ludwig-Maximilians-University of Munich, Munich, Germany

Carolina Maruta - Lisbon Faculty of Medicine, Language Research Laboratory, Lisbon, Portugal Simon Mead - MRC Prion Unit, Department of Neurodegenerative Disease, UCL Institute of Neurology, Queen Square, London, UK

Lieke Meeter - Department of Neurology, Erasmus Medical Center, Rotterdam, Netherlands 
. Gabriel Miltenberger - Faculty of Medicine, University of Lisbon, Lisbon, Portugal

- Rick van Minkelen - Department of Clinical Genetics, Erasmus Medical Center, Rotterdam, The Netherlands

Sara Mitchell - LC Campbell Cognitive Neurology Research Unit, Sunnybrook Research Institute, Toronto, Canada

- Benedetta Nacmias - Department of Neuroscience, Psychology, Drug Research and Child Health, University of Florence, Florence, Italy

. Mollie Neason - Department of Neurodegenerative Disease, UCL Institute of Neurology, London, UK

Jennifer Nicholas - The London School of Hygiene and Tropical Medicine, London, UK Linn Öijerstedt - Division of Neurogeriatrics, Karolinska Institutet, Stockholm, Sweden Jaume Olives - Alzheimer's disease and other cognitive disorders unit, Neurology Department, Hospital Clinic, Institut d'Investigacions Biomèdiques, Barcelona, Spain Jessica Panman - Department of Neurology, Erasmus Medical Center, Rotterdam, The Netherlands Janne Papma - Department of Neurology, Erasmus Medical Center, Rotterdam, The Netherlands Maximilian Patzig - Department of Neuroradiology, Ludwig-MaximiliansUniversity Munich, Germany

Michela Pievani - Istituto di Ricovero e Cura a Carattere Scientifico Istituto Centro San Giovanni di Dio Fatebenefratelli, Brescia, Italy

Yolande Pijnenburg - VUMC, Amsterdam, The Netherlands

Sara Prioni - Fondazione Istituto di Ricovero e Cura a Carattere Scientifico Istituto Neurologico Carlo Besta, Milan, Italy

Catharina Prix - Department of Neurology, Ludwig-Maximilians-University Munich, Germany Rosa Rademakers - Department of Neurosciences, Mayo Clinic, Jacksonville, Florida, USA 
- Veronica Redaelli - Fondazione Istituto di Ricovero e Cura a Carattere Scientifico Istituto Neurologico Carlo Besta, Milan, Italy

. Tim Rittman - University of Cambridge, Cambridge, UK

- Ekaterina Rogaeva - Tanz Centre for Research in Neurodegenerative Diseases, University of

Toronto, Toronto, Canada

Pedro Rosa-Neto - Translational Neuroimaging Laboratory, McGill University Montreal, Québec,

Canada

- Giacomina Rossi - Fondazione Istituto di Ricovero e Cura a Carattere Scientifico Istituto

Neurologico Carlo Besta, Milan, Italy

Martin Rossor - Department of Neurodegenerative Disease, UCL Institute of Neurology, London,

UK

- Beatriz Santiago - Neurology Department, Centro Hospitalar e Universitário de Coimbra, Coimbra, Portugal

Elio Scarpini - University of Milan, Centro Dino Ferrari, Milan, Italy; Fondazione IRCSS Ca' Granda, Ospedale Maggiore Policlinico, Neurodegenerative Diseases Unit, Milan, Italy

Elisa Semler - Ulm University, Ulm, Germany

Rachelle Shafei - Department of Neurodegenerative Disease, UCL Institute of Neurology, London, UK

Christen Shoesmith - Department of Clinical Neurological Sciences, University of Western Ontario, London, Ontario, Canada

Miguel Tábuas-Pereira - Centre of Neurosciences and Cell Biology, Universidade de Coimbra, Coimbra, Portugal

Mikel Tainta - Neuroscience Area, Biodonostia Health Research Institute, Paseo Dr Begiristain sn, CP 20014, San Sebastian, Gipuzkoa, Spain 
. David Tang-Wai - University Health Network Memory Clinic, Toronto Western Hospital, Toronto,

\section{Canada}

. David L Thomas - Neuroradiological Academic Unit, UCL Institute of Neurology, London, UK

- Hakan Thonberg - Center for Alzheimer Research, Division of Neurogeriatrics, Karolinska Institutet, Stockholm, Sweden

. Carolyn Timberlake - University of Cambridge, Cambridge, UK

Pietro Tiraboschi - Fondazione Istituto di Ricovero e Cura a Carattere Scientifico Istituto Neurologico Carlo Besta, Milano, Italy

Philip Vandamme - Neurology Service, University Hospitals Leuven, Belgium; Laboratory for Neurobiology, VIB-KU Leuven Centre for Brain Research, Leuven, Belgium

. Mathieu Vandenbulcke - Geriatric Psychiatry Service, University Hospitals Leuven, Belgium;

Neuropsychiatry, Department of Neurosciences, KU Leuven, Leuven, Belgium

Michele Veldsman - University of Oxford, UK

Ana Verdelho - Department of Neurosciences, Santa Maria Hospital, University of Lisbon, Portugal Jorge Villanua - OSATEK Unidad de Donostia, San Sebastian, Gipuzkoa, Spain

Jason Warren - Department of Neurodegenerative Disease, UCL Institute of Neurology, London, UK

. Carlo Wilke - Hertie Institute for Clinical Brain Research, University of Tuebingen, Tuebingen, Germany

Henrik Zetterberg - Department of Neurodegenerative Disease, UCL Institute of Neurology, London, UK

Miren Zulaica - Neuroscience Area, Biodonostia Health Research Institute, Paseo Dr Begiristain sn, CP 20014, San Sebastian, Gipuzkoa, Spain 


\section{REFERENCES}

1 Rascovsky K, Hodges JR, Knopman D, et al. Sensitivity of revised diagnostic criteria for the behavioural variant of frontotemporal dementia. Brain. 2011;134:2456-77.

2 Gorno-Tempini ML, Hillis AE, Weintraub S, et al. Classification of primary progressive aphasia and its variants. Neurology. 2011;76:1006-14.

3 Deleon J, Miller BL. Frontotemporal dementia. Handb Clin Neurol. 2018;148:409-30.

4 Van Deerlin VM, Sleiman PM, Martinez-Lage M, et al. Common variants at 7p21 are associated with frontotemporal lobar degeneration with TDP-43 inclusions. Nat Genet. 2010;42:234-9.

5 Lattante S, Le Ber I, Galimberti D, et al. Defining the association of TMEM106B variants among frontotemporal lobar degeneration patients with GRN mutations and C9orf72 repeat expansions. Neurobiol Aging. 2014;35:2658 e1- e5.

6 Pottier C, Zhou X, Perkerson RB 3rd, et al. Potential genetic modifiers of disease risk and age at onset in patients with frontotemporal lobar degeneration and GRN mutations: a genome-wide association study. Lancet Neurol. 2018;17:548-58.

7 Zhang M, Ferrari R, Tartaglia MC, et al. A C6orf10/LOC101929163 locus is associated with age of onset in C9orf72 carriers. Brain. 2018;141:2895-907.

8 Stern Y. Cognitive reserve in ageing and Alzheimer's disease. Lancet Neurol. 2012;11:1006-12.

9 Borroni B, Premi E, Agosti C, et al. Revisiting brain reserve hypothesis in frontotemporal dementia: evidence from a brain perfusion study. Dement Geriatr Cogn Disord. 2009;28:130-5. 10 Premi E, Grassi M, van Swieten J, et al. Cognitive reserve and TMEM106B genotype modulate brain damage in presymptomatic frontotemporal dementia: a GENFI study. Brain. 2017;140:178491.

11 Wang HX, MacDonald SW, Dekhtyar S, et al. Association of lifelong exposure to cognitive reserve-enhancing factors with dementia risk: A community-based cohort study. PLoS Med. 
2017;14:e1002251.

12 Stern Y, Gurland B, Tatemichi TK, et al. Influence of education and occupation on the incidence of Alzheimer's disease. JAMA. 1994;271:1004-10.

13 Foubert-Samier A, Catheline G, Amieva H, et al. Education, occupation, leisure activities, and brain reserve: a population-based study. Neurobiol Aging. 2012;33:423 e15-25.

14 Sole-Padulles C, Bartres-Faz D, Junque C, et al. Brain structure and function related to cognitive reserve variables in normal aging, mild cognitive impairment and Alzheimer's disease. Neurobiol Aging. 2009;30:1114-24.

15 Nyberg L, Lovden M, Riklund K, et al. Memory aging and brain maintenance. Trends Cogn Sci. 2012;16:292-305.

16 Stern Y. An approach to studying the neural correlates of reserve. Brain Imaging Behav. 2017;11:410-6.

17 Rohrer JD, Nicholas JM, Cash DM, et al. Presymptomatic cognitive and neuroanatomical changes in genetic frontotemporal dementia in the Genetic Frontotemporal dementia Initiative (GENFI) study: a cross-sectional analysis. Lancet Neurol. 2015;14:253-62.

18 Premi E, Calhoun VD, Diano M, et al. The inner fluctuations of the brain in presymptomatic Frontotemporal Dementia: The chronnectome fingerprint. Neuroimage. 2019;189:645-654. 19 Tabrizi SJ, Langbehn DR, Leavitt BR, et al. Biological and clinical manifestations of Huntington's disease in the longitudinal TRACK-HD study: cross-sectional analysis of baseline data. Lancet Neurol. 2009;8:791-801.

20 Bateman RJ, Xiong C, Benzinger TL, et al. Clinical and biomarker changes in dominantly inherited Alzheimer's disease. N Engl J Med. 2012;367:795-804.

21 Schneider R, McKeever P, Kim T, et al. Downregulation of exosomal miR-204-5p and miR-632 as a biomarker for FTD: a GENFI study. J Neurol Neurosurg Psychiatry. 2018;89(8):851-858. 
22 Desmarais P, Rohrer JD, Nguyen QD, et al. Therapeutic trial design for frontotemporal dementia and related disorders. J Neurol Neurosurg Psychiatry. 2019;90(4):412-423.

23 Gazzina S, Manes MA, Padovani A, et al. Clinical and biological phenotypes of frontotemporal dementia: Perspectives for disease modifying therapies. Eur J Pharmacol. 2017;817:76-85.

24 Hamalainen S, Joutsa J, Sihvonen AJ, et al. Beyond volume: A surface-based approach to bilingualism-induced grey matter changes. Neuropsychologia. 2018;117:1-7.

25 Sun J, Chen Q, Zhang Q, et al. Training your brain to be more creative: brain functional and structural changes induced by divergent thinking training. Hum Brain Mapp. 2016;37:3375-87. 26 Rehfeld K, Luders A, Hokelmann A, et al. Dance training is superior to repetitive physical exercise in inducing brain plasticity in the elderly. PLoS One. 2018;13:e0196636.

27 Hill NT, Mowszowski L, Naismith SL, et al. Computerized Cognitive Training in Older Adults With Mild Cognitive Impairment or Dementia: A Systematic Review and Meta-Analysis. Am J Psychiatry. 2017;174:329-40.

28 Leung IH, Walton CC, Hallock $\mathrm{H}$, et al. Cognitive training in Parkinson disease: A systematic review and meta-analysis. Neurology. 2015;85:1843-51.

29 Teixeira CVL, Ribeiro de Rezende TJ, Weiler M, et al. Cognitive and structural cerebral changes in amnestic mild cognitive impairment due to Alzheimer's disease after multicomponent training. Alzheimers Dement (N Y). 2018;4:473-80.

30 Harding SR, Bocchetta M, Gordon E, et al. The TMEM106B risk allele is associated with lower cortical volumes in a clinically diagnosed frontotemporal dementia cohort. J Neurol Neurosurg Psychiatry. 2017; 88(11):997-998.

31 Valenzuela MJ, Sachdev P, Wen W, et al. Lifespan mental activity predicts diminished rate of hippocampal atrophy. PLoS One. 2008;3:e2598.

32 Suo C, Leon I, Brodaty $\mathrm{H}$, et al. Supervisory experience at work is linked to low rate of 
hippocampal atrophy in late life. Neuroimage. 2012;63:1542-51.

33 Lo RY, Jagust WJ, Alzheimer's Disease Neuroimaging I. Effect of cognitive reserve markers on Alzheimer pathologic progression. Alzheimer Dis Assoc Disord. 2013;27:343-50.

34 Cabeza R, Albert M, Belleville $S$, et al. Maintenance, reserve and compensation: the cognitive neuroscience of healthy ageing. Nat Rev Neurosci. 2018;19:701-10.

35 Almeida RP, Schultz SA, Austin BP, et al. Effect of Cognitive Reserve on Age-Related Changes in Cerebrospinal Fluid Biomarkers of Alzheimer Disease. JAMA Neurol. 2015;72:699-706.

36 Sheikh-Bahaei N, Sajjadi SA, Pierce AL. Current Role for Biomarkers in Clinical Diagnosis of Alzheimer Disease and Frontotemporal Dementia. Curr Treat Options Neurol. 2017;19:46.

37 Pham TM, Ickes B, Albeck D, et al. Changes in brain nerve growth factor levels and nerve growth factor receptors in rats exposed to environmental enrichment for one year. Neuroscience. 1999;94:279-86.

38 Naka F, Narita N, Okado N, et al. Modification of AMPA receptor properties following environmental enrichment. Brain Dev. 2005;27:275-8.

39 Kempermann G, Gast D, Gage FH. Neuroplasticity in old age: sustained fivefold induction of hippocampal neurogenesis by long-term environmental enrichment. Ann Neurol. 2002;52:135-43. 40 Rampon $\mathrm{C}$, Jiang $\mathrm{CH}$, Dong $\mathrm{H}$, et al. Effects of environmental enrichment on gene expression in the brain. Proc Natl Acad Sci U S A. 2000;97:12880-4.

41 Ekstrand J, Hellsten J, Tingstrom A. Environmental enrichment, exercise and corticosterone affect endothelial cell proliferation in adult rat hippocampus and prefrontal cortex. Neurosci Lett. 2008;442:203-7.

42 Placek K, Massimo L, Olm C, et al. Cognitive reserve in frontotemporal degeneration: Neuroanatomic and neuropsychological evidence. Neurology. 2016;87:1813-9. 43 Premi E, Gualeni V, Costa P, et al. Looking for Measures of Disease Severity in the 
Frontotemporal Dementia Continuum. J Alzheimers Dis. 2016;52:1227-35.

44 Bandres-Ciga S, Noyce AJ, Hemani G, et al. Shared polygenic risk and causal inferences in amyotrophic lateral sclerosis. Ann Neurol. 2019;85:470-481. 


\section{FIGURE LEGENDS}

\section{Figure 1. Model design of Structural Equation Model.}

The explanatory variable is enclosed in the blue box, while response variables in green (grey matter volume) and pink (Mini-mental examination test) circles. For convenience the indicator variables, covariates and error terms are not displayed. An arrow from one variable to another indicates that the first variable has a causal influence on the latter.

Grey arrows indicate the tested effect of education on cognitive performances and grey matter volumes; orange arrows indicate the tested effect of grey matter volumes on cognitive performances at each time point; purple arrows indicate the tested effects of baseline measures on slopes' measures; blue arrows indicate the tested interaction effects between slopes and baseline measures (see Methods for details).

MMSE: Mini-mental examination test; GMV: grey matter volume; i: intercept; s: slope.

\section{Figure 2. Significant results from the Structural Equation Model.}

Significant effects in either mutation carriers and non-carriers are depicted by dark grey arrows, while significant effects only in mutation carriers are depicted by light grey arrows (see Results section for details).

MMSE: Mini-mental examination test; GMV: grey matter volume; i: intercept; s: slope. 